\title{
Die moderne Impulsoszillometrie im Spektrum lungenfunktioneller Messmethoden
}

\author{
Modern Impulse Oscillometry in the Spectrum of Pulmonary Function \\ Testing Methods
}

Autoren

Institute
J. Winkler ${ }^{1}$, A. Hagert-Winkler ${ }^{2}$, H. Wirtz ${ }^{3}$, G. Hoheisel ${ }^{1}$

Fachpraxen für Pneumologie und Allergologie, Leipzig

Klinik und Poliklinik für Diagnostische Radiologie, Klinikum der Universität Leipzig AöR

3 Medizinische Klinik und Poliklinik I, Klinikum der Universität Leipzig AöR eingereicht 5.2.2009

akzeptiert nach Revision 4. 6.2009

\section{Bibliografie}

DOI 10.1055/s-0029-1214938

Pneumologie 2009; 63:

461-469 @ Georg Thieme

Verlag KG Stuttgart · New York

ISSN 0934-8387

\section{Korrespondenzadresse}

Dr. med. Jörg Winkler

Praxis für Pneumologie/

Allergologie

Kreuzstraße 2a

04103 Leipzig

Lungewinkler@aol.com

\section{Zusammenfassung \\ $\nabla$}

Die Impulsoszillometrie (IOS) ist eine computergestützte Methode zur Messung komplexer mechanischer Eigenschaften der Atemwege. Eine obstruktive Atemwegserkrankung kann durch die IOS insgesamt sicher diagnostiziert werden, sofern bei schwerer Obstruktion komplexe IOSParameter wie Frequenzabhängigkeit der Resistance (FDR) und niederfrequente Reactance (X5) mit einbezogen werden. Die Parameter der Standardverfahren Pneumotachografie, Spirometrie und Bodyplethysmografie zeigen aufgrund unterschiedlicher Messprinzipien relativ geringe statistische Korrelationen zur IOS wie auch bekanntermaßen untereinander, begründet in unterschiedlichen Messprinzipien. Die IOS ist eine patientenschonende und wenig kooperationsabhängige Methode zur Evaluierung obstruktiver Atemwegserkrankungen, die bisherige Standardmethoden sinnvoll ergänzen, jedoch nicht ersetzen kann und die sich darüber hinaus als sensitives Screeninginstrument zur frühzeitigen Erkennung einer Bronchialobstruktion nicht nur in der Pädiatrie und der Arbeitsmedizin eignet.

\section{Einleitung}

$\nabla$

Aufgrund der Häufigkeit und Progredienz der chronisch obstruktiven Lungenerkrankung (COPD) und der weiter steigenden Prävalenz des Asthma bronchiale sind die Ansprüche an die Verfügbarkeit und Sensitivität der Diagnostik gewachsen. Gleichzeitig ist das ökonomische Spannungsfeld bezüglich Investitionskosten und messtechnischem Aufwand schärfer geworden [1]. Eine modernen Ansprüchen genügende Lungenfunktionsdiagnostik sollte deshalb bei vertretbarem technischem Aufwand die wesentlichen

Unserem verehrten klinischen Lehrer Professor Dr. Joachim Schauer zum 70. Geburtstag gewidmet.

\section{Abstract \\ $\nabla$}

Impulse oscillometry (IOS) is a computer supported method for the measurement of complex mechanical airway characteristics. Bronchial obstructions can reliably be diagnosed by IOS when more complex parameters such as frequency dependence of resistance (FDR) and lung reactance (X5) are evaluated in cases with more severe obstruction. Parameters of conventional methods like pneumotachography, spirometry, and bodyplethysmography show relatively low correlations due to the different measurement principles, not only in comparison to IOS, but also in between one another. The IOS is a convenient method for patients with a low dependency on cooperation for the evaluation of obstructive airway diseases complementary to the established standard methods. Furthermore, IOS may provide a sensitive screening tool for the early detection of bronchial obstructions not only in paediatrics but also in occupational medicine.

Komponenten der Obstruktion bei geringer Kooperationsabhängigkeit und Patientenbelastung erfassen und im Idealfall ortsvariabel (z. B. am Arbeitsplatz) einsetzbar sein. Zur Messung des Strömungswiderstandes, des Einsekundenvolumens (FEV1) und weiterer Parameter, die eine Atemwegsobstruktion definieren, stehen heute verschiedene Methoden wie die Spirometrie/Pneumotachografie die Atemwiderstandsmessung mittels Unterbrechermethode, die Ganzkörperplethysmografie und in zunehmendem Maße auch die Methode der Impulsoszillometrie (IOS) zur Verfügung [2-9]. Die IOS ist jedoch ein „Stiefkind“ der standardisierten Lungenfunktionstestungen und wird vergleichsweise wenig eingesetzt. Dies liegt nicht zuletzt an der Tatsache, 
dass die Methode eine gegenüber Standardverfahren komplexere Interpretationsweise erfordert. Ein weiterer Grund für die zurückhaltende Anwendung der Technik dürfte in der Unsicherheit in Bezug auf die Vergleichbarkeit zu den Standardmethoden der Lungenfunktionsdiagnostik liegen. Der vorliegende Artikel diskutiert Grundlagen und Literaturdaten zum Verständnis und zur Einordnung der IOS ergänzend zu einer kürzlich erschienenen Originalarbeit [10], ohne den Anspruch auf eine abschließende Bewertung zu erheben. Bezüglich weitergehender Informationen zur Standarddiagnostik wird auf kürzlich erschienene Leitlinien und Publikationen verwiesen [11-13].

\section{Impulsoszillometrie (IOS) \\ $\nabla$}

Bei dem Prinzip der IOS erfolgt eine aktive Energiezufuhr in Form von impulsförmigen Schwingungen (Oszillationen), die durch einen externen Generator erzeugt und der Ruheatmung des Patienten überlagert werden, während dieser über ein Mundstück und einen definierten Abschlusswiderstand Raumluft atmet. Die Reaktion des respiratorischen Systems auf die Druckimpulse wird analysiert, indem die am Mundstück entstehende Impulsströmung sowie der resultierende Impulsdruck registriert werden. Der so ermittelte komplexe Atemwiderstand (Impedanz Zrs) wird gegenüber dem bodyplethysmografisch bestimmten Atemwegswiderstand Rtot etwas höher gemessen, da die Impedanz Teilwiderstände beinhaltet, die sich hinsichtlich ihres zeitlichen Zusammenhanges zwischen Druck und Fluss unterscheiden [14-17] und die über eine reine Querschnittsbeschreibung der Atemwege hinausgehende Informationen liefern:

1. Resistance (R), Reibungswiderstand (Strömungswiderstand, auch Wirkwiderstand genannt) in den Atemwegen.

2. Reactance $(X)$, auch Blindwiderstand unterteilt in:

a) inertive Reactance (Xin), auch als Inertance bezeichnet, (beinhaltet alle trägen Widerstände innerhalb des Lunge-Thoraxsystems einschließlich der Masseträgheit der ventilierten Luftsäule), b) kapazitive Reactance (Xcap) auch als elastischer oder complianter Widerstand (Capacitance) bezeichnet, bei der Kompression von Gasen (Volumencompliance) als auch bei der Überwindung von elastischen Dehnungswiderständen (Membrancompliance) auftretend.

Vereinfachend wird die Reactance bei $5 \mathrm{~Hz}$ wegen der Widerspiegelung von pulmonalen Gewebseigenschaften auch als Lungenreactance bezeichnet.

Die IOS mit computergestützter Auswertung ist eine Weiterentwicklung der polifrequenten oszillometrischen Verfahren und stellt die derzeit modernste Variante der multifrequenten Oszil- lationsmethoden dar [17]. Mittels IOS werden Merkmale des untersuchten respiratorischen Systems in Form eines komplexen Impedanzspektrums wiedergegeben und die Komponenten der registrierten Impedanz (Resistance $\mathrm{R}$, Reactance $\mathrm{X}$ ) in ihrer Frequenzabhängigkeit dargestellt, wobei eine hohe zeitliche Auflösung durch Applikation von in der Regel 5 Messimpulsen pro Sekunde erreicht wird ( $\bullet$ Abb. 1). Jeder Impuls hat dabei einen multifrequenten Inhalt $[17,19,20]$.

Die Resistance ( $R$ ) wird bei 5 und $20 \mathrm{~Hz}$ (R5, R20) ausgewertet, die Reactance $(X)$ bei $5 \mathrm{~Hz}$ (X5) [17]. Dabei repräsentiert R5 vorwiegend Informationen über die gesamten Atemwege. Durch absolute und relative Differenzbildung zum höherfrequent gemessenen Widerstand R20 läßt sich die Frequenzabhängigkeit der Resistance $(F D R)$ charakterisieren $(F D R$ absolut $=$ R5 - R20, FDR relativ $=($ R5 - R20)/R20) [10].

Die Reactance X5 als Maß für die elastischen (kapazitativen) und volumeninhärenten Eigenschaften der Lunge wird hauptsächlich von peripheren obstruktiven Veränderungen und Wandeigenschaften der Bronchien beeinflusst. Sollwertüberschreitungen um mindestens 30-50\% werden als pathologisch angesehen. Grundsätzlich sind die Kategorien zentral und peripher nicht statisch morphologisch, sondern funktionell und variabel zu verstehen.

Der totale Atemwiderstand R5, die Reactance X5, die Resonanzfrequenz der Atemwege Fres sowie der spektrale Verlauf von Resistance und Reactance (Frequenzabhängigkeit) können neben der Einschätzung des Schweregrades der Obstruktion auch zu deren funktioneller Differenzierung in zentrale und periphere Komponenten herangezogen werden ( $\bullet$ Abb. 2). Die Resonanzfrequenz kann auch als indirektes Maß für einen Abfall (eine Negativierung) des Parameters X5 betrachtet werden. So findet sich bei vorwiegend peripherer Obstruktion und pathologisch erniedrigter Lungenreactance eine sich in stärkerem Maße negativ ausprägende Reactance X5 und folglich eine höhere Resonanzfrequenz [18]. Auch die Resistancewerte zeigen bei dieser Konstellation eine deutlichere Frequenzabhängigkeit mit höheren Werten bei niedrigen Frequenzen (R5 >> R20). Die standardisierte (relative) Frequenzabhängigkeit FDR und die absolute Frequenzabhängigkeit der Resistance FDRa werden als sensible Indikatoren der peripheren Atemwegsobstruktion diskutiert [21]. Unter Zugrundelegung von Literaturangaben [22] und von mittleren Normwerten für R5 (z.B. 0,34 kPa/l/s) und R20 (z. B. 0,29 kPa/l/s) ergibt sich rechnerisch ein erwarteter Wert für FDR von $0,16 \pm 0,02 \mathrm{kPa} /$ $1 / s$, entsprechend einem oberen Grenzwert $(x+2 s)$ von etwa $0,20 \mathrm{kPa} / \mathrm{l} / \mathrm{s}$. Höhere Werte der FDR signalisieren eine größere Differenz zwischen R5 und R20, bezogen auf den Absolutwert von R20, also eine stärkere Frequenzabhängigkeit der oszillatori-

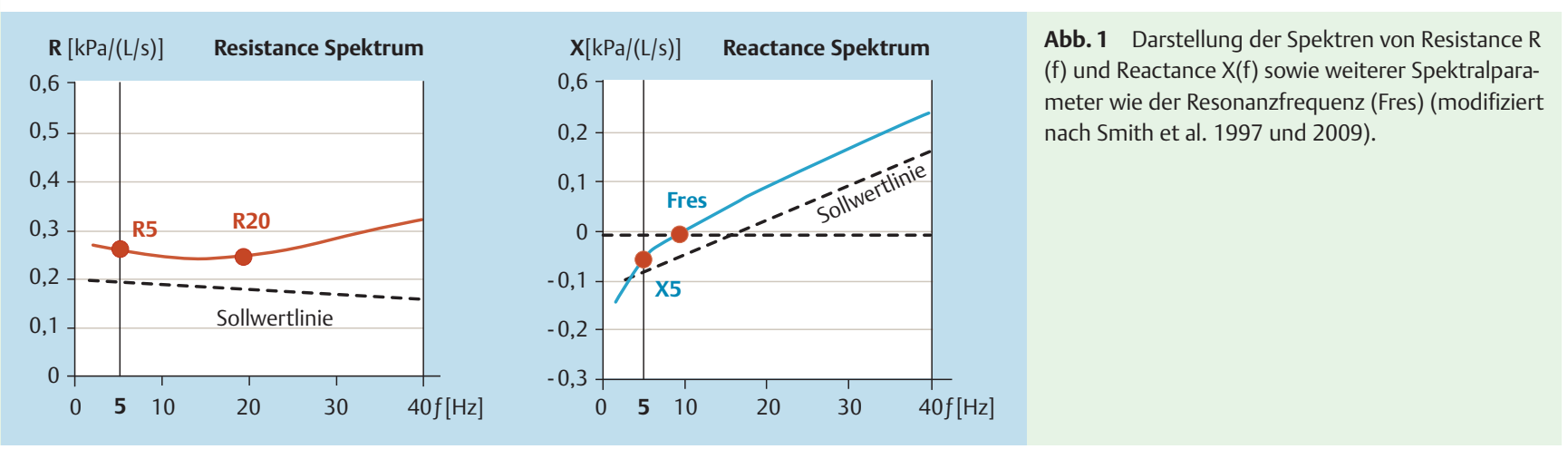




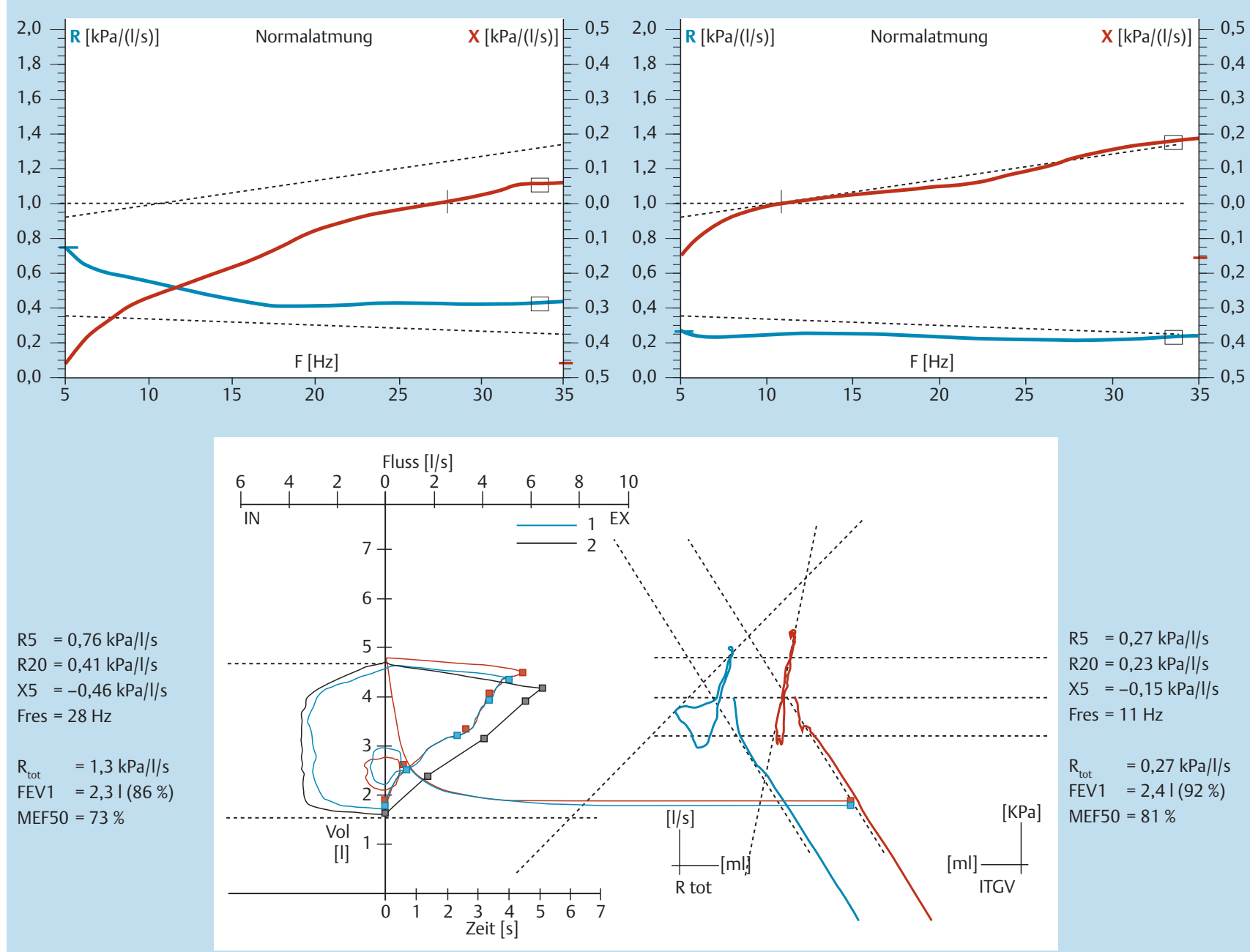

Abb. 2 Verläufe der IOS-Spektralparameter R und X mit Charakterisierung der Fres im Vergleich zur Standardmethodik am Beispiel einer 41-jährigen Patientin mit Asthma bronchiale vor und nach Bronchospasmolysetestung mit Salbutamol-Dosier-Aerosol. Die überwiegend periphere Obstruktion bei Ruheatmung mit Besserung nach Spasmolyse wird durch die Pneumotachografie im Gegensatz zu Bodyplethysmografie und IOS nicht adäquat dargestellt bei unveränderter Atemmittellage (Werte nicht dargestellt). Die gestrichelten Linien zeigen Sollbereiche (blau - Resistance, rot - Reactance).
Impulsoszillometrisch zeigt sich der Bronchospasmolyseeffekt bezüglich der Resistance (blaue durchgezogene Linie) durch eine Reduktion besonders im niederfrequenten Bereich (Rückgang R5 > R20) und durch eine Verminderung der Frequenzabhängigkeit von $\mathrm{R}$ (Steigung zwischen 20 und $5 \mathrm{~Hz}$ ). Bezüglich der Reactance (Blindwiderstände) zeigt sich ein geringerer Betrag der niederfrequenten (negativen) Kapazität (X5-Verschiebung in Richtung Null) und ein sehr deutlicher Rückgang der Resonanzfrequenz (Linksverschiebung vertikaler schwarzer Strich). schen Resistance. Weitere Sollwertstudien unter Einbeziehung repräsentativer Altersstichproben besonders zu den FDR-Parametern sind aus Sicht der Autoren analog zur Sollwertdiskussion bei den Standardmethoden [11] sinnvoll.

Periphere Obstruktionen, deren Erfassung mittels stärker flussabhängiger Messverfahren wie der Pneumotachografie limitiert ist, sind zusammenfassend oszillometrisch durch einen erhöhten totalen Atemwiderstand (R5), einen stärker frequenzabhängigen Resistanceverlauf mit Anstieg der FDR (absolut und relativ), eine Abnahme der Lungenreactance X5 sowie eine Zunahme der Resonanzfrequenz Fres gekennzeichnet. Zentrale Obstruktionen sind hingegen durch einen im Normbereich liegenden Reactanceverlauf $X(f)$, eine nahezu normale Fres und einen vergleichbar zu R5 erhöhten R20 sowie durch eine niedrige FDR charakterisiert [18].

Die IOS erlaubt bei geringem apparativem Aufwand eine fortlaufende Widerstandsbestimmung bei nur geringen Anforderungen an die Mitarbeit des Patienten [23-26]. Als besonders nützlich hat sich die IOS wegen der minimalen Mitarbeitsabhängigkeit und der geringen Patientenbelastung auch bei jüngeren Kindern erwiesen [27-36].

\section{Vergleich der IOS zu den Standardmethoden \\ $\nabla$}

Die bronchiale Strömungsbehinderung lässt sich durch Messung des Einsekundenvolumens (FEV1) bei forcierter Atmung spirometrisch quantifizieren, wobei für den Nachweis einer Obstruktion die relative Einsekundenkapazität (Tiffeneau-Index, FEV1\% VCin) die spezifischere Messgröße darstellt. Das FEV1 zeigt bei COPD in der Regel eine nur geringe, bei Asthma hingegen eine größere Variabilität $[37,38]$. Das FEV1 ist neben der Patientenmitarbeit besonders bei COPD jedoch stark von der Stabilität der peripheren Atemwege abhängig und erlaubt keine eindeutige Differenzierung zwischen peripherer und zentraler Obstruktion. Die Elastizität der Atemwege ist bei COPD vermindert und führt bei forcierter Atmung zur exspiratorischen Kollapsneigung mit teils fixierter Flussbegrenzung [39,40]. Darin liegt neben der 
starken Mitarbeitsabhängigkeit eine methodische Begrenzung der Diagnostik chronisch obstruktiver Atemwegserkrankungen mittels Spirometrie [12,41-43].

Weitere Parameter der forcierten Spirometrie sind der expiratorische Spitzenfluss (Peak Expiratory Flow, PEF) und die mittleren exspiratorischen Flusswerte (MEF). Die MEF bei 75, 50 und 25\% der Vitalkapazität (MEF75, MEF50, MEF25) gelten im Gegensatz zum FEV1 und PEF als weniger mitarbeitsabhängig und werden teilweise als Hinweis auf eine periphere Atemwegsobstruktion („small airways disease“) benutzt [12,43-46]. Nachteilig ist die hohe Variabilität dieser Parameter. Leichte Obstruktionen können sowohl in der Spirometrie, z. B. beim Asthmatiker, atemmuskulär kompensiert, also quasi „überblasen“ werden [40-42]. Die nach peripher sich verringernde Strömungsgeschwindigkeit bei zunehmendem Gesamtquerschnitt der Bronchien („Weibelsches Trompetenmodell der Atemwege“) führt außerdem dazu, dass bei Schädigung der kleinen Atemwege die Flussparameter an Sensitivität verlieren („quiet zone“).

Die Ganzkörperplethysmografie (Bodyplethysmografie) gilt als Goldstandard der Atemwegswiderstands- und Lungenvolumenmessung und erlaubt bei Ruheatmung u.a. die Bestimmung des intrathorakalen Gasvolumens (ITGV bzw. der funktionellen Residualkapazität FRC), des Residualvolumens (RV) über das ITGV sowie des spezifischen Atemwegswiderstands z.B. als sRtot, sRaw und daraus abgeleitet als Rtot bzw. Raw [13,46-48]. Als grundsätzliche Eigenschaft der Atemwegswiderstände ist festzuhalten, dass sie im Wesentlichen die Obstruktionen der zentralen Atemwege $[46,49]$ widerspiegeln. Eine keulenförmige Deformierung der Rtot-Kurve spricht für eine Phasenverschiebung zwischen Alveolardruck und Atemfluss im Sinne einer inhomogenen Obstruktion („air trapping“). Die primär gemessene spezifische Resistance (sRaw) in ihrer Wechselwirkung aus Resistance und intrathorakalem Gasvolumen berücksichtigt Verfälschungen durch eine veränderte Atemmittellage und verbessert die Sensitivität und Reproduzierbarkeit der bodyplethysmografischen Untersuchung. Nachteile der Bodyplethysmografie sind der apparative und methodische Aufwand, hohe Kosten und die eingeschränkte Einsetzbarkeit bei Kleinkindern, in der Neonatologie, Anästhesiologie, Intensivmedizin oder am Arbeitsplatz [47,50,51]. Besonders die Messung des ITGV weist eine Mitarbeits- und damit Störanfälligkeit auf [13].

Bezüglich der Messparameter werden FEV1 und sRtot bzw. sReff in der Routine als Standard für die Erfassung bronchialer Obstruktionen angesehen [13,52 - 54]. Trotz der erkennbar geringeren Sensitivität wird die höhere Spezifität der Flussparameter als Vorteil gesehen, was sich international auch in der Betonung des FEV1 als Leitparameter in den GOLD-Richtlinien [52] und des PEF in den GINA-Leitlinien ausdrückt [12]. Die Beobachtung, dass bei nicht wenigen Patienten eine Obstruktion bzw. eine bronchiale Hyperreaktivität nur bodyplethysmografisch erkannt wird, spricht jedoch gegen die alleinige Anwendung der FEV1-Messung und für den Einsatz von Widerstandsmessverfahren unter Ruheatmung und damit auch für die sensitive Resistancemessung mittels IOS $[10,13,46,55]$.

Bei der Messung der Atemwegswiderstände zeigt sich die Sensitivität der bodyplethysmografisch bestimmten totalen Resistance (Rtot) gegenüber der spezifischen Resistance (sRtot) statistisch erwartungsgemäß unterlegen [10]. Dieser Unterschied lässt sich durch die oben geschilderte Berücksichtigung der Lungenüberblähung im Parameter sRtot erklären, da mit höherer Atemmittellage eine mechanische Vordehnung der kleinen Atemwege erfolgt und somit mittels Rtot scheinbar zu niedrige Widerstandswerte gemessen werden [13].

Als Alternativtechnik zur direkten und wenig fluss- und mitarbeitsabhängigen Widerstandsmessung bei Ruheatmung bietet sich die moderne IOS an. Bisherige Arbeiten beziehen sich häufig auf Kinder [27,56 - 58]. Bei der Bewertung oszillometrischer Obstruktionsparameter werden spirometrisch ermittelte Parameter und bodyplethysmografisch gemessene Resistancewerte als Referenzverfahren eingesetzt $[10,27,59-66]$, was wegen der unterschiedlichen physikalischen Messprinzipien allerdings nur begrenzt sinnvoll ist. Abb. 2 zeigt den Verlauf der IOS-Spektralparameter R und X mit Charakterisierung der Fres im Vergleich zu Befunden der Spirometrie/Pneumotachografie und Bodyplethysmografie am Beispiel einer normalgewichtigen Patientin vor und nach Bronchospasmolystest zur Illustration der sensitiven Erfassung peripherer und die Lungencompliance betreffender Veränderungen durch IOS und Bodyplethysmografie.

Unterschiede zwischen oszillometrischen und ganzkörperplethysmografisch bestimmten Messparametern sind dennoch bekannt und auch zu erwarten $[60,67,68]$. So werden gering höhere Normwertgrenzen für den oszillometrischen Atemwiderstand gegenüber dem bodyplethysmografisch gemessenen Atemwegswiderstand angegeben [14,17]. Der Parameter R5 wird als gering höher gegenüber Rtot beschrieben [10,63]. Der mittlere Raw wird ebenfalls kleiner gemessen als die oszillometrischen Resistancewerte bei niedrigen Frequenzen [10]. Bereits früh wurde darauf hingewiesen, dass eine Erhöhung von Rtot wesentlich aus der Einengung der zentralen Atemwege resultiert [49]. Die IOS vermag, bedingt durch das wenig flussabhängige Messprinzip, periphere Obstruktionen der kleinen Atemwege unter Einbeziehung von mechanischen Wandeigenschaften der Atemwege sensitiver zu erfassen und kann Atemwegswiderstände von der Lungenreactance partiell unterscheiden [22,62]. Die bisher beobachtete hohe Sensitivität der komplexen IOS-Parameter birgt zwar die grundsätzliche Gefahr von „falsch positiven“ Werten der Oszillometrie, denen „normale“ bodyplethysmografische Resistancewerte im Sinne einer geringeren Spezifität („Überschätzung leichter Obstruktionen“) gegenüberstehen, kann jedoch für Screening- und Frühdiagnostikverfahren Vorteile bieten.

Mittels IOS bestimmte Resistancewerte liegen häufig sowohl bei höheren Messfrequenzen als auch bei schwerer Obstruktion deutlich unter den bodyplethysmografischen Resistancewerten, wodurch es zu einer scheinbaren „Unterschätzung“ schwerer Obstruktionen kommen kann $[60,63,67,69]$. Die diagnostische Information einer Atemwegsobstruktion geht mit steigendem Obstruktionsgrad zunehmend auf die Lungenreactance X5 über, sodass diese bei Befundinterpretationen ( $\bullet$ Tab. 1) immer gleichrangig zu den reinen Resistancewerten beachtet werden sollte. Mit dem Kombinationsparameter Impedanz Zrs, der die Komponenten $\mathrm{R}$ und $\mathrm{X}$ einbezieht, ist eine bessere Einschätzung schwerer Obstruktionen gegenüber der alleinigen Resistance R5 folgerichtig zu erwarten $[60,69]$.

Da neben den beiden Impedanzkomponenten $\mathrm{R}$ und $\mathrm{X}$ diagnostische Informationen über Art und Ausmaß einer Atemwegsobstruktion im gesamten von der Oszillationsmethode erfassten Frequenzverlauf vorhanden sind, werden Vorteile von formspezifischen Kennwerten erwartet, welche die Frequenzabhängigkeit von $\mathrm{R}(\mathrm{f})$ und $\mathrm{X}(\mathrm{f})$ quantitativ charakterisieren. Schon der Verlauf des $R(f)-S p e k t r u m s$ erlaubt eine Trennung der Resistance in einen funktionell zentralen und peripheren Anteil und liefert somit detaillierte Aussagen über eine Obstruktion. Auch die von der Lungenreactance abhängige Resonanzfrequenz Fres wird als 
Tab. 1 Vorschlag zur Schweregradeinteilung der Obstruktion durch gemeinsame Interpretation von Resistance und Reactance anhand von Sollwertabweichungen (nach Smith 2008, pers. Mitteilung), zur Vereinfachung wird in der 1. Zeile ein Berechnungsbeispiel auf Grundlage eines X5 Sollwertes von - 0,15 dargestellt.

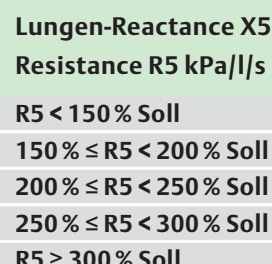

R5 $\geq 300 \%$ Soll

X5 $<$ (X5 Soll - 0,15)
z. B. $<-0,30$
normal
I (mild)
II (mittel)
III (schwer)
IV (sehr schwer)

X5 $<$ (X5 Soll - 0,3)
z. B. $<-0,45$
I (mild)
II (mittel)
III (schwer)
IV (sehr schwer)
IV (sehr schwer)

X5 < X5 Soll -0,45)
Z. B. $<-0,60$
II (mittel)
III (schwer)
IV (sehr schwer)
IV (sehr schwer)
IV (sehr schwer)

\author{
X5 $<($ X5 Soll $-0,60)$ \\ Z. B. $<-0,75$ \\ III (schwer) \\ IV (sehr schwer) \\ IV (sehr schwer) \\ IV (sehr schwer) \\ IV (sehr schwer)
}

empfindlicher Parameter für die Charakterisierung einer Obstruktion bei gemeinsamer Interpretation mit der Resistance beschrieben $[19,62]$.

Die Parameter Fres und X5 zeigen im klinischen Einsatz im Gegensatz zu R5 signifikante Unterschiede zwischen allen Schweregradgruppen der Obstruktion [10]. Zum Obstruktionsnachweis durch oszillatorisch bestimmte Widerstandsparameter zeigen insbesondere niedrige Messfrequenzen eine gute Empfindlichkeit. Die höherfrequente IOS Resistance (R20) leistet als Einzelparameter zwar keinen signifikanten Beitrag zur Schweregradabschätzung der Obstruktion, ermöglicht aber die Berechnung der Frequenzabhängigkeit der Resistance [10,60,62,69-72]. Die mit stärkerer Obstruktion zunehmende Frequenzabhängigkeit des RSpektrums lässt sich durch den Parameter FDR, welcher bisher kein IOS-Routineparameter ist, einfach quantifizieren [10,59, $60,73,74]$. Ein hoher FDR-Wert charakterisiert eine größere Steilheit des Resistance-Spektrums, bedingt durch höhere R5 und/oder niedrigere R20-Werte. Gerade diese Frequenzabhängigkeit der Resistance wird als guter Marker für das Ausmaß der Bronchokonstriktion angesehen $[23,66,75]$. Die Frequenzabhängigkeit wird darüber hinaus als sensitiver Indikator für das Vorliegen einer frühen peripheren Atemwegsobstruktion gedeutet $[21,69,76]$. In einer vergleichenden Untersuchung an gesunden und symptomarmen asthmatischen Kindern erwies sich die FDR als die am besten trennende Messgröße [73]. Neben X5 und Fres weist nur die FDR signifikante Unterschiede zwischen allen Schweregradgruppen der Obstruktion auf und liegt bezogen auf die Sollwertabweichung trotz höherer Streuungen in allen Obstruktionsgraden der Rtot am nächsten [10]. Die Abgrenzung mittelgradiger gegenüber leichtgradigen Obstruktionen erscheint mit verschiedenen IOS-Parametern außer R20 gut möglich.

Für eine bessere Aussagekraft der komplexeren IOS-Parameter spricht deren gegenüber R5 deutlichere Korrelation zu Rtot und FEV1 [10]. Die insgesamt relativ geringen statistischen Korrelationen zwischen oszillometrischer einerseits und pneumotachografischen und bodyplethysmografischen Parametern andererseits werden durch die beschriebenen messmethodischen Unterschiede erklärt und zeigen die Erfassung von unterschiedlichen Aspekten der Atemwegsobstruktion sowie von Wandeigenschaften durch die verschiedenen Methoden [10,22,53,60,61, $63,67,69,77-79]$. Bei der klassischen Oszillometrie wurden für Normalpersonen in einzelnen Untersuchungen auch höhere Korrelationen zwischen Ros bei $6 \mathrm{~Hz}$ (FOT) und Raw beschrieben [12].

Ein direkter Vergleich von Korrelationskoeffizienten weiterer IOS-Parameter zu Standardmethoden ist wegen der starken Abhängigkeit vom Schweregrad der Obstruktion und Einflüsse restriktiver Faktoren besonders auf die IOS limitiert. In einer Studie mit einer gemischten obstruktiv-restriktiven Patientengruppe finden sich die höchsten Korrelationen von Rtot zu Fres, Zrs, X5 und zu R5 [61]. Für die Widerstandswerte bei höheren Frequenzen fanden sich geringere bzw. keine signifikanten Korrelationen zum bodyplethysmografischen Widerstand. Korrelationen $\mathrm{zu}$ den exspiratorischen Flusswerten (MEF) wurden für R5 und für Zrs berichtet [61]. Zwischen dem FEV1 und Parametern der multifrequenten Oszillometrie wie R8, X8 und Fres wurden Korrelationskoeffizienten von unter 0,3 beschrieben $[77,80]$. Bei einer größeren Untersuchung an 216 Asthmapatienten lagen die Korrelationen von R5 und Zrs zum FEV1 bei -0,49 [81], eine andere Studie bei jugendlichen Asthmatikern beschreibt recht enge Korrelationen zwischen FEV1 und R5, X5 sowie der absoluten Frequenzabhängigkeit FDR aus R5 und R15 [59].

Trotz insgesamt niedriger Korrelationskoeffizienten für die IOSMethode konnte eine Korrelation zur Pneumotachografie für X5, Fres und FDR gezeigt werden [10,80-82]. Insgesamt erscheinen die Korrelationen von Parametern der IOS zur Bodyplethysmografie jedoch deutlicher als zur Pneumotachografie/Spirometrie. Unter den IOS-Parametern zeigen die FDR und die Fres, gefolgt von X5, R5 und der diese beiden Parameter beinhaltenden Impedanz Zrs eine der bodyplethysmografischen Resistance vergleichbare, gegenüber den reinen Flussparametern jedoch höhere Empfindlichkeit.

Die Spezifität pathologischer IOS-Frühparameter sollte trotz der gut belegten physiologischen Grundlagen und der der vorliegenden Literaturdaten im Einzelfall immer durch weitergehende Untersuchungen wie Bodyplethysmografie und Provokationstestung abgesichert werden, um falsch positive Befunde auszuschließen, was für die Eignung der IOS für Screeninguntersuchungen aus Sicht der Autoren jedoch keinen entscheidenden Nachteil darstellt.

\section{Klinischer Nutzen der IOS}

Die Spirometrie bzw. Pneumotachografie ist technisch wenig aufwendig, weist aber einen erheblichen Mitarbeitsbedarf seitens des Patienten auf. Bei bestehender bronchialer Kollapsneigung besteht durch das unphysiologische forcierte Exspirationsmanöver die Gefahr der Überschätzung von Obstruktionen bzw. der Unterschätzung von Therapieeffekten. Außerdem bestehen Zweifel an der Korrelation der FEV1-Befunde zum klinischen Dyspnoeempfinden des Patienten und zur körperlichen Belastbarkeit [83]. Frühformen der Obstruktion werden möglicherweise darüber hinaus durch muskuläre Kompensation beim forcierten Atemmanöver nicht optimal erfasst.

Die Bodyplethysmografie ist zwar noch immer der Goldstandard für die Messung des Atemwegswiderstandes und der Lungenvolumina, differenziert aber Veränderungen der pathophysiologisch bedeutsamen kleinsten Atemwege [46] bei atemzugbasier- 


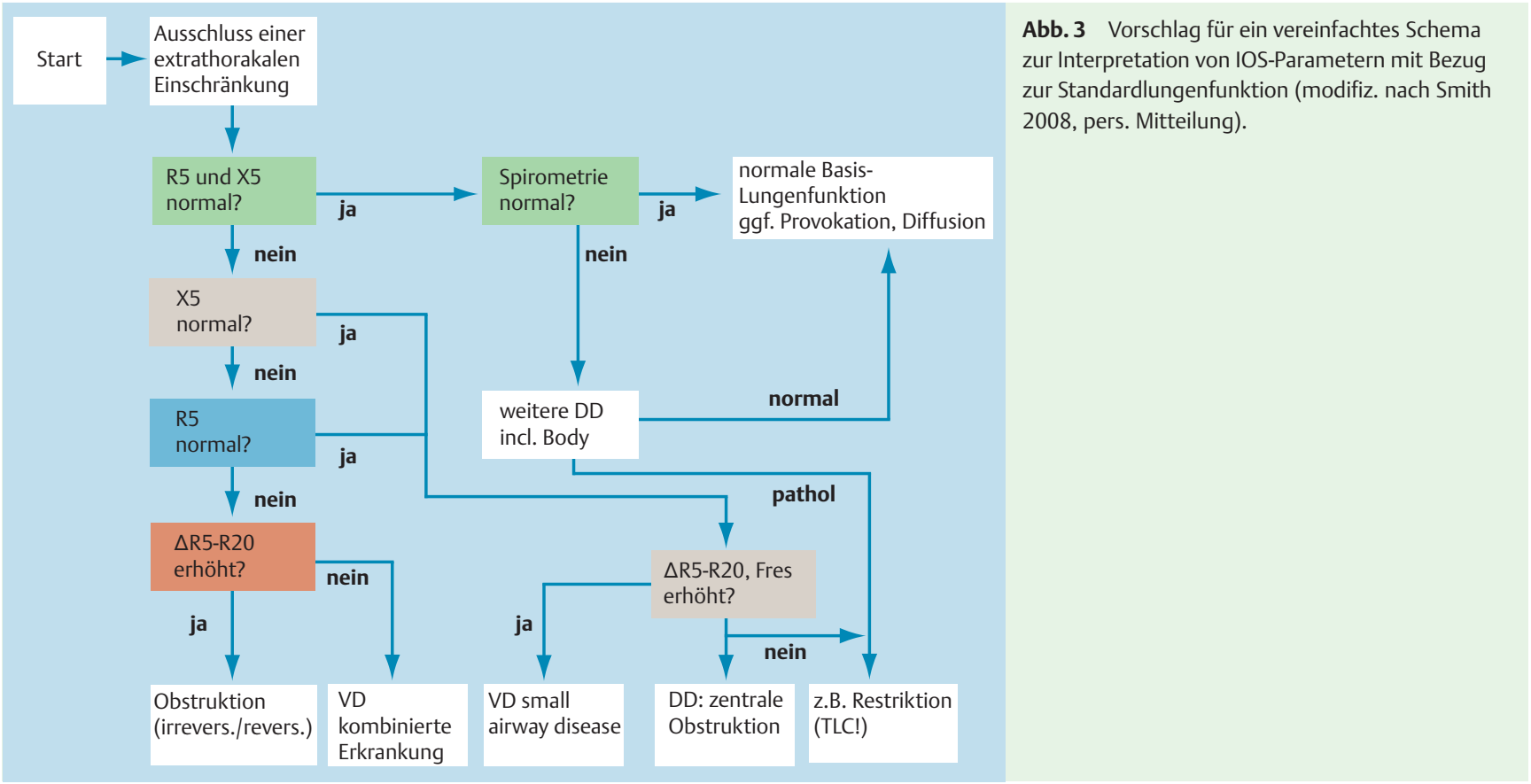

tem Messprinzip nicht optimal. Nachteile der Bodyplethysmografie sind der hohe technische Aufwand, hohe Kosten und die fehlende Mobilität des Messsystems.

Für die Untersuchung mittels IOS, die auch in einer portablen Variante verfügbar ist, genügen wenige normale Ruheatemzüge, wobei der zeitliche Aufwand in der Regel unter dem der Standardmethoden liegt [1]. Dies zeigt auch die sehr gute praktische Durchführbarkeit bei älteren Patienten und im häuslichen Milieu $[80,84]$. Artefakte können durch ungleichmäßige Atmung, Schluckakte und inkorrekten Lippenschluss sowie fehlende Stützung der Wangen entstehen. Vorteilhaft ist die serielle Bestimmung der Einzelwiderstände entlang des Tracheobronchialbaumes sowohl numerisch als auch anhand einer bildlichen Darstellung. Damit kann sich der Untersucher die Ergebnisse der Resistance- und Reactance-Spektren trotz der komplizierten mathematischen Grundlagen grafisch veranschaulichen [1,17]. Die Vielzahl ableitbarer IOS-Parameter erfordert eine Gewichtung, wobei sich insgesamt höhere Messwertstreuungen gegenüber den Standardparametern zeigen können [10]. Als empfindliche Obstruktionsparameter mit vergleichsweise guter Korrelation zur Bodyplethysmografie erweisen sich wie oben ausgeführt die Lungenreactance (X5), der oszillatorische Widerstand (R5), die Resonanzfrequenz (Fres) und Frequenzabhängigkeit des Resistancespektrums (FDR) [10]. Ein Schema zur Erleichterung der komplexen Interpretation im Zusammenhang mit der Standardlungenfunktion wird in $\mathbf{A b b} \mathbf{3}$ vorgeschlagen, ohne Anspruch auf Vollständigkeit und Berücksichtigung aller möglichen Konstellationen erheben zu können.

Alle drei diskutierten Messverfahren erfassen unterschiedliche funktionelle Komponenten, wodurch eine direkte Vergleichbarkeit oder auch Ersetzbarkeit erschwert wird. Die IOS misst über die oben genannten Faktoren mit Einbeziehung elastischer Wandeigenschaften eine zusätzliche Komponente der Bronchialobstruktion und kann damit nicht primär eine der Standardmethoden ersetzen. Ein Nachteil der IOS gegenüber der Bodyplethysmografie stellt die fehlende Erfassung von Lungenvolumina, besonders des Residualvolumens dar. Bei restriktiven Störungsmustern wird die ungenaue Abgrenzung mit Auswirkungen auf die IOS-Widerstandsparameter kritisch beschrieben $[61,66]$. Die Schwächen der klassischen Oszillometrie mit Überschätzung leichter und Unterschätzung schwerer Obstruktionen sind bei Verwendung geeigneter und komplexer Parameter deutlich geringer ausgeprägt.

Die IOS hat aufgrund ihrer hohen Empfindlichkeit und der sehr guten Handhabbarkeit das Potenzial einer aussagekräftigen, wenig aufwendigen Vorfeldmethode, mit der sich bereits beginnende, klinisch noch nicht manifeste Obstruktionen sensitiver als mit der Spirometrie bzw. Pneumotachografie allein nachweisen lassen, wodurch interessante Einsatzgebiete bei Risikopatienten und bei der Indikationsstellung für bronchiale Provokationstestungen erschlossen werden. Besonders die Parameter X5 und Fres sowie abgeleitet Zrs erfassen zusätzlich erhöhte Blindwiderstände, die auf ein verändertes bronchopulmonales Trägheitsund Elastizitätsverhalten und somit strukturelle Veränderungen (Remodelling) hinweisen. Vergleichende Daten an größeren Patientengruppen zwischen Standardmethoden und IOS zur Frühund Screeningdiagnostik wären von hohem wissenschaftlichen Wert zur fundierten Standortbestimmung beider Methoden in diesem Indikationsgebiet. Die Nachteile oszillometrischer Messverfahren bei der Erfassung schwerer Obstruktionen können durch die abgeleiteten komplexeren Parameter bei höheren Messwertstreuungen teilweise ausgeglichen werden.

Zusammenfassend ergeben sich für die IOS aus Sicht der Autoren derzeit folgende sinnvolle Einsatzbereiche:

1. Messungen bei eingeschränkter Kooperationsfähigkeit, wie z. B. bei Kindern, Schwerkranken und Älteren [1,27 - 36, 82]. Durch geeignete Vorrichtungen sind auch spezielle IOS-Einsatzmöglichkeiten, einschließlich Untersuchungen am Krankenbett in Anästhesie und Intensivmedizin durchführbar geworden.

2. Die schweregradabhängige Empfindlichkeit der Methode schafft die Voraussetzung für einen Einsatz im Screening, so bei Risikogruppen (z. B. Rauchern, nach Inhalationstraumen bei Umweltkatastrophen), am Arbeitsplatz (z. B. Bäckerasthma) sowie in der Erkennung der belastungsinduzierten Obstruktion z.B. nach Ergometrie [4,62, 85-88]. 
3. Die IOS ist zur Verlaufskontrolle obstruktiver Lungenerkrankungen grundsätzlich geeignet, wobei höhere Schweregrade besser durch die Parameter Fres, X5 und Zrs als durch R5 allein beschrieben werden [10]. Ein interessanter Zusatzparameter ist die Frequenzabhängigkeit des Atemwegswiderstandes (FDR), während von einer isolierten Interpretation von R20 keine wesentlichen Aussagen zu erwarten sind. Insgesamt wird in der Literatur selbst bei Kindern eine gute Reproduzierbarkeit der Impulsoszillometrie-Messparameter beschrieben $[27,57-59]$. Untersuchungen zu häuslichen Selbstmessungen mit tragbaren oszillatorischen Messsystemen zeigten eine gute Praktikabilität und Messgenauigkeit für den oszillatorischen Widerstand [84].

4. Das IOS-Messverfahren kann die koventionellen Verfahren der Lungenfunktionsdiagnostik ergänzen und zusätzlich weniger flussabhängige und die peripheren Atemwege betreffende Krankheitskomponenten besser erfassen und die Sensitivität besonders der Spirometrie erhöhen [84]. Die Trennschärfe der Bodyplethysmografie für verschiedene Obstruktionsschweregrade und die geringe Streubreite der Spirometrie mit kleinen Variationskoeffizienten für das FEV1 $[27,89]$ sowie die Erfassung wesentlicher Lungenvolumina sprechen jedoch für die Beibehaltung der Standardverfahren. Bei Nichtverfügbarkeit der Bodyplethysmografie ist die IOS als Ergänzung zur Spirometrie/Pneumotachografie empfehlenswert [78].

\section{Schlussfolgerung}

Die IOS als computergestützte Weiterentwicklung der klassischen oszillometrischen Atemwiderstandsmessung ist wegen ihrer komplexen, über die reine Widerstands- bzw. Flussmessung hinausgehenden Aussagekraft, die auch strukturelle Eigenschaften der Atemwege wie Lungenkapazität, Trägheitseigenschaften und elastische Rückstellkräfte mit einbezieht, von hohem wissenschaftlichem und klinischem Interesse. Hinzu kommen vergleichsweise geringe Anschaffungskosten, niedrige Ansprüche an die Kooperation des Patienten und ein geringer Messaufwand mit Eignung auch für Kinder, ältere Personen und bettseitig $[1,80,84]$. Die Befundinterpretation erfordert eine gewisse Erfahrung, die über eine einfache Analogie zu bodyplethysmografisch gemessenen Atemwegswiderständen hinausgeht ( $\bullet$ Tab. 1 und - Abb. 2). Sensitive Abweichungen von IOS-Frühparametern bedürfen einer Spezifitätsprüfung mittels Standardmethodik bis hin zur Provokationstestung. Die IOS ist trotz ihrer komplexen Interpretationsmodelle als praktikable, patienten- und bedienerfreundliche Methode anzusehen, die komplementär zu den Standardmethoden weitere Aspekte der Atemwegsobstruktion erfasst und sich gut für den Einsatz auch in der pneumologischen Praxis eignet.

Die Autoren danken Herrn Smith aus Würzburg sehr herzlich für seine konstruktiven und kompetenten Hinweise anlässlich der Revision des Artikels.

\section{Interessenkonflikte}

Für alle Autoren besteht kein Interessenkonflikt.

\section{Literatur}

1 Vogel J, Smith HJ. Entwicklungsstand der Impulsoszillometrie 2003. Pneumologie 2003; 57: 763 -764

2 Aronsson D, Tufvesson E, Ankerst J et al. Allergic rhinitis with hyper-responsiveness differ from asthma in degree of peripheral obstruction during metacholine challenge test. Clin Physiol Funct Imaging 2008; 28: $81-85$

3 Park JW, Lee YW, Jung YH et al. Impulse oscillometry for estimation of airway obstruction and bronchodilation in adults with mild obstructive asthma. Ann Allergy Asthma Immunol 2007; 98: 546-552

4 Oppenheimer BW, Goldring RM, Herberg ME et al. Distal airway function in symptomatic subjects with normal spirometry following World Trade Center dust exposure. Chest 2007; 132: 1275-1282

5 Nguyen TU, Diong B, Nazeran $\mathrm{H}$ et al. A study of IOS data using two mead-related models of respiratory impedance. Conf Proc IEEE Eng Med Biol Soc 2007; 2007: 1078-1081

6 Al-Mutairi SS, Sharma PN, Al-Alawi A et al. Impulse oscillometry: an alternative modality to the conventional pulmonary function test to categorise obstructive pulmonary disorders. Clin Exp Med 2007; 7: 56 64

7 Singh D, Tal-Singer R, Faiferman I et al. Plethysmography and impulse oscillometry assessment of tiotropium and ipratropium bromide; a randomized, double-blind, placebo-controlled, cross-over study in healthy subjects. Br J Clin Pharmacol 2006; 61: 398 - 404

8 Houghton CM, Woodcock AA, Singh D. A comparison of plethysmography, spirometry and oscillometry for assessing the pulmonary effects of inhaled ipratropium bromide in healthy subjects and patients with asthma. Br J Clin Pharmacol 2005; 59: $152-159$

9 Shiota S, Katoh M, Fujii M et al. Predictive equations and the reliability of the impulse oscillatory system in Japanese adult subjects. Respirology $2005 ; 10$ : $310-315$

10 Winkler J, Hagert-Winkler A, Wirtz H et al. Die Impulsoszillometrie in der Diagnose obstruktiver Lungenerkrankungen. Pneumologie 2009; 63: $266-275$

11 Marek W, Marek E, Mückenhoff K et al. Lungenfunktion im Alter: Brauchen wir neue Referenzwerte? Pneumologie 2009; 63: 235-243

12 Criée CP, Berdel D, Heise D et al. Empfehlungen der Deutschen Atemwegsliga zur Spirometrie. Oberhaching: Dustri Verlag, 2006

13 Criée CP, Berdel D, Heise D et al. Empfehlungen der Deutschen Atemwegsliga und der Gesellschaft für Pneumologie und Beatmungsmedizin zur Ganzkörperplethysmographie. Bad Lippspringe: Deutsche Atemwegsliga, Oberhaching: Dustri, 2009

14 Nussbaum E, Galant SP. Measurement of total respiratory resistance in children by a modified forced oscillation method. Pediatr Res 1984; 18: $139-145$

15 Korn V, Franetzki M, Prestele K. A simplified approach to the measurement of respiratory impedance. Prog Resp Res 1979; 11: 144-161

16 Vogel J, Nolte D, Smith HJ. Workshop Impulsoszillometrie Bad Reichenhall. München: Dustri, 1995

17 Vogel J, Smidt U. Impulsoszillometrie. Analyse der Atemmechanik in Ambulanz und Klinik, Epidemiologie und experimenteller Forschung. Frankfurt/M: pmi, 1994

18 Smith HJ, Vogel J, Arnold J et al. Impuls-Oszillometrie in der Früherkennung obstruktiver Atemwegserkrankungen. Sonderausgabe IOS, Firma Jaeger. Höchberg, 1997

19 Fichter J, Wilkens JH, Fabel H. Die oszillatorische Messung des Atemwiderstands mit dem custo vit im Vergleich zum Oszillaire und zur Bodyplethysmografie. Pneumologie 1989; 43: 382 - 386

20 Holle JP, Landser F, Schüller B et al. Measurement of Respiratory Mechanics with forced Oscillations. Comparison of Two Methods (Siregnost FD 5 versus Pseudorandom noise technique). Respiration 1981; 41: $119-127$

21 Clement J, Landser FJ, van de Woestijne KP. Total resistance and reactance in patients with respiratory complaints with and without airway obstruction. Chest 1983; 83: 215-220

22 Wouters EFM. Total respiratory impedance measurement by forced oscillations: a non-invasive method to assess bronchial response in occupational medicine. Exp Lung Res 1990; 16: 25 - 40

23 Brochard L, Pelle G, de Palmas J et al. Density and frequency dependence of resistance in early airway obstruction. Am Rev Respir Dis 1987; 135: $579-584$

24 Cuijpers CE, Wesseling GJ, Kessels AG et al. Low diagnostic value of respiratory impedance measurements in children. Eur Respir J 1997; 10: 88-93 
25 Glaser T, Pleger E, Müller E et al. Erprobungsbericht zur polyfrequenten Oszilloresistometrie mit dem Gerät custo vit. Pneumologie 1989; 43: $369-375$

26 Peslin R, Felicio da Silva J, Duvivier C et al. Respiratory mechanics studied by forced oscillations during artificial ventilation. Eur Respir J 1993; 6: $772-784$

27 Bisgaard H, Klug B. Lung function measurement in awake young children. Eur Respir J 1995; 8: 2067 - 2075

28 Frey $U$, Silverman $M$, Kraemer $R$ et al. High-frequency Respiratory Impedance Measured by Forced-Oscillation Technique in Infants. Am J Respir Crit Care Med 1998; 158: 363 - 370

29 Hall GL, Hantos Z, Wildhaber JH et al. Methacholine responsiveness in infants assessed with low frequency forced oscillation and forced expiration techniques. Thorax 2001; 56: $42-47$

30 Malmberg LP, Mäkelä MJ, Mattila PS et al. Exercise-induced changes in respiratory impedance in young wheezy children and nonatopic controls. Pediatr Pulmonol. 2008; 43: 538- 44

31 Song TW, Kim KW, Kim ES et al. Correlation between spirometry and impulse oscillometry in children with asthma. Acta Paediatr 2008; 97: $51-54$

32 Nieto A, Pamies $R$, Oliver $F$ et al. Montelukast improves pulmonary function measured by impulse oscillometry in children with asthma (Mio study). Respir Med 2006; 100: 1180-1185

33 Tomalak W, Radliński J, Pawlik J et al. Impulse oscillometry vs. body plethysmography in assessing respiratory resistance in children. Pediatr Pulmonol 2006; 41: 50-54

34 Frei J, Jutla J, Kramer G et al. Impulse oscillometry: reference values in children 100 to $150 \mathrm{~cm}$ in height and 3 to 10 years of age. Chest 2005; 128: $1266-1273$

35 Olaguíbel JM, Alvarez-Puebla MJ, Anda M et al. Comparative analysis of the bronchodilator response measured by impulse oscillometry (IOS), spirometry and body plethysmography in asthmatic children. J Investig Allergol Clin Immunol 2005; 15: $102-106$

36 Marotta A, Klinnert MD, Price MR et al. Impulse oscillometry provides an effective measure of lung dysfunction in 4-year-old children at risk for persistent asthma. J Allergy Clin Immunol 2003; 112: 317 - 322

37 ATS, American Thoracic Society. Standards for the diagnosis and care of patients with chronic obstructive pulmonary disease (COPD) and asthma. Am J Respir Crit Care Med 1995; 152: 77-120

38 Lange P, Parner J, Vestbo P et al. A 15-year follow-up study of ventilatory function in adults with asthma. N Engl J Med 1998; 339: 11941200

39 Saetta M, Finkelstein R, Cosio MG. Morphological and cellular basis for airflow limitation in smokers. Eur Respir J 1994; 7: 1505-1515

40 Thurlbeck WM. Pathology of chronic airflow obstruction. Chest 1990; 97: $6 s-10 s$

41 O'Donnell DE, Sanii $R$, Anthonisen NR et al. Effect of dynamic airway compression on breathing pattern and respiratory sensation in severe chronic obstructive pulmonary disease. Am Rev Respir Dis 1987; 135: $912-918$

42 Smith HR, Irvin CG, Cherniack RM. The utility of spirometry in the diagnosis of reversible airways obstruction. Chest 1992; 101: 1577-1581

43 Macklem PT. Obstruction in small airways-a challenge to medicine. Am Med 1972; 52: 721 - 724

44 Murray AB, Ferguson AC. A comparison of spirometric measurements in allergen bronchial challenge testing. Clin Allergy 1981; 11: 87-93

45 Orehek J. Measurement of airway hyperresponsiveness in man. Eur J Resp Dis 1982; 117: 42-61

46 Seidel A. Atemstoßtest, Atemgrenzwert, Resistance und Fluß-Volumenkurve - Möglichkeiten einer umfassenden Charakterisierung der Atemwegsobstruktion und der Atemreserve. Pneumologie 1998; 52: $437-438$

47 Nolte D. Einführung zum IOS-Workshop Bad Reichenhall. In: Vogel J, Nolte D, Smith HJ, Hrsg Workshop Impuls-Oszillometrie Bad Reichenhall. Oerhaching: Dustri Verlag, 1995

48 Dubois AB, Bothelo SY, Comroe JH. A new method for measuring airway resistance in man using a bodyplethysmograph: Values in normal subjects and in patients with respiratory disease. J Clin Invest 1956; 35 : $327-335$

49 Schnellbächer $F$. Lungenfunktionsprüfungen mit der einfachen und erweiterten Oszilloresistometrie, Schriftenreihe Zentralblatt für Arbeitsmedizin, Arbeitschutz, Prophylaxe und Ergonomie 8. Heidelberg: Dr. Curt Haefner, 1981

50 Kanengiser S, Dozor AJ. Forced expiratory maneuvers in children aged 3 to 5 years. Pediatr Pulmonol 1994; 18: $144-149$
51 Sly PD, Robertson CF. A review of pulmonary function testing in children. J Asthma 1990; 27: 137-147

52 GOLD . Global strategy for the diagnosis, management and prevention of chronic obstructive pulmonary disease (updated 2007). National institutes of health. www.goldcopd.com; Stand: 2007

53 van Noord JA, Smeets J, Clement J et al. Assessment of reversibility of airflow obstruction. Am J Crit Care Med 1994; 150: 551 - 554

54 Wise RA, Connett J, Kurnow K et al. Selection of spirometric measurements in a clinical trial, the Lung Health Study. Am J Respir Crit Care Med 1995; 151: 675-681

55 Taube C, Lehnigk B, Paasch Ket al. Factor analysis of changes in dyspnea and lung function parameters after bronchodilatation in chronic obstructive pulmonary disease. Am J Respir Crit Care Med 2000; 162: $216-220$

$56 \mathrm{Klug}$ B, Bisgaard $\mathrm{H}$. Measurement of lung function in awake 2-4-yearold asthmatic children during methacholine challenge and acute asthma: a comparison of the impulse oscillation technique, the interrupter technique, and transcutaneous measurement of oxygen versus wholebody plethysmography. Pediatr Pulmonol 1996; 21: 290 - 300

57 Malmberg LP, Pelkonen A, Poussa T et al. Determinants of respiratory system input impedance and bronchodilator response in healthy Finnish preschool children. Clin Physiol Funct Imaging 2002; 22: 64-71

58 Ortiz G, Menendez $R$. The effects of inhaled albuterol and salmeterol in 2 - to 5-year old asthmatic children as measured by impulse oscillometry. J Asthma 2002; 39: 531 - 536

59 Goldmann MD, Carter R, Klein R et al. Within- and between- day variability of respiratory impedance, using impulse oscillometry in adolescent asthmatics. Pediatr Pulmonol 2002; 34: 312-319

60 Müller E, Harzendorf E, Merker G et al. Theoretische und experimentelle Untersuchungen zur diagnostischen Relevanz und Vergleichbarkeit oszillationsmechanischer Parameter. Pneumologie 1989; 43: 339-347

61 Schmidt $U$. Untersuchungen zum Stellenwert der Impulsoszillometrie in der Diagnostik restriktiver Lungenfunktionsstörungen. Medizinische Dissertation. Berlin 1998

62 Kohlhäufl M, Brand P, Scheuch G et al. Impulse oscillometry in healthy nonsmokers and asymptomatic smokers: effects of bronchial challenge with methacholine. J Aerosol Med 2001; 14: 1-12

63 Hellinckx J, Cauberghs M, De Boeck K et al. Evaluation of impulse oscillation system: comparison with forced oscillation technique and body plethysmography. Eur Respir J 2001; 18: 564 - 570

64 Nielsen KG, Bisgaard H. Discriminative capacity of bronchodilator response measured with three different lung function techniques in asthmatic and healthy children aged 2 to 5 years. Am J Respir Crit Care Med 2001; 164: 554-559

65 Wesseling GJ, Vanderhoven-Augustin IM, Wouters EF. Forced oscillation technique and spirometry in cold air provocation tests. Thorax 1993; 48: $254-259$

66 van Noord JA, Clement J, van de Woestijne KP et al. Total respiratory resistance and reactance in patients with asthma, chronic bronchitis and emphysema. Am Rev Respir Dis 1991; 143: 922 - 927

67 Petro W, Loytved G, Korn V et al. Inhalativer bronchialer Provokationstest - diagnostische Aussagekraft verschiedener Funktionsmethoden (Spirografie, Pneumotachografie, Bodyplethysmografie, Oszillometrie). Prax Klin Pneumol 1983; 37: 85-90

68 Smith HJ, Vogel J. Praktische Umsetzung der Impuls-Oszillometrie Stand 2003. Pneumologie 2003; 57: 765

69 Landser FJ, Nagels J, Demedts $M$ et al. A new method to determine frequency charakteristics of the respiratory system. J Appl Physiol 1976; 41: $101-106$

70 Buhr W, Jörres R, Berdel D et al. Correspondence between forced oscillation and body plethysmography during bronchoprovokation with carbachol in children. Pediatr Pulmonol 1990; 8: 280-288

71 Sixt R, Aronsson PH, Solymar L. The forced oscillation technique in children - with special reference to the use of low frequencies. Eur Respir Rev 1991; 1: 222-225

72 Wuthe H, Uhrner R, Kleckow $M$ et al. Verbesserung der Atemwiderstandsmessung durch Senkung der oszillatorischen Frequenz. Prax Klin Pneumol 1988; 42: 790-792

73 Buhr W, Landser F, Reimnitz P et al. Lungenfunktionsuntersuchungen an gesunden und symptomarmen asthmakranken Kindern im Alter von 5 bis 16 Jahren. Atemwegs-Lungenkrankh 1987; 13: 370-374

74 Pasker HG, Mertens I, Clement J et al. Normal values of total respiratory input resistance and reactance for adult men and women. Eur Respir Rev 1994; 4: $134-137$ 
75 Delacourt C, Lorino H, Herve-Guillot $M$ et al. Use of the forced oscillation technique to assess airway obstruction and reversibility in children. Am J Crit Care Med 2000; 161: 730 - 736

76 Kjeldgaard JM, Hyde RW, Speers DM et al. Frequency dependence of total respiratory resistance in early airway disease. Am Rev Respir Dis 1976; 114: $501-508$

77 Wesseling GJ, Wouters EF. Respiratory impedance measurements in a dose-response study of isocapnic hyperventilation with cold air. Respiration 1992; 59: 259-264

78 Delacourt C, Lorino H, Fuhrmann C et al. Comparision of the forced oscillation technique and the interrupter technique for assessing airway obstruction and its reversibility in children. Am J Respir Crit Care Med 2001; 164: 965 - 972

79 Klein G. Kontinuierliche Messungen des Atemwiderstands (Ros, Ru) bei bronchialen Provokations- und Spasmolysetests. Atemwegs-Lungenkrankh 1995; 21: 199-205

80 Janssens JP, Nguyen MC, Herrmann FR et al. Diagnostic value of respiratory impedance measurements in elderly subjects. Respir Med 2001; 95: $415-422$

$81 \mathrm{Kim} \mathrm{CW}$, Kim JS, ParkJW et al. Clinical applications for forced oscillation techniques (FOT) in patients with bronchial asthma. Korean J Intern Med 2001; 16: 80-86

82 Vink GR, Arets HG, van der Laag J et al. Impulse oscillometry: a measure for airway obstruction. Pediatr Pulmonol 2003; 35: 214-219
83 Taube C, Jörres RA, Magnussen H. Akute Effekte von Bronchodilatatoren auf Lungenfunktion und Dyspnoe bei Patienten mit COPD. Pneumologie 2000; 54: 252 - 255

84 Rigau J, Burgos F, Hernandez C et al. Unsupervised self-testing of airway obstruction by forced oscillation at patients home. Eur Respir J 2003; 22: $668-671$

85 Becher G, Beck E, Rothe $M$ et al. Untersuchungen mit der Impulsoszillometrie (IOS) an Lungengesunden unter Feldbedingungen - Reproduzierbarkeit und Altersregression. Pneumologie 1998; 52: 69-0

86 Eichler R, Vogel J, Arnold J et al. Optionen der impulsoszillometrischen Analyse in der ambulanten, klinischen und experimentellen Lungenfunktionsdiagnostik Entwicklungsergebnisse 1992/93. Pneumologie 1994; 48: 852

87 Goldman M, Knudson RJ, Mead J et al. A simplified measurement of respiratory resistance by forced oscillation. J Appl Physiol 1970; 28: $113-116$

88 Marotta A, Klinnert MD, Price MR et al. Impulse oscillometry provides an effective measure of lung dysfunction in 4-year-old children at risk for persistent asthma. J Allergy Clin Immunol 2003; 112: 317-322

89 Kastelik JA, Aziz I, Ojoo JC et al. Evaluation of impulse oscillation system: comparision with forced oscillation technique and body plethysmography. Eur Respir J 2002; 19: 1214-1219 